\title{
Women architects on the web
}

\author{
Florencia Marciani ${ }^{1}$ e Inés Moisset ${ }^{2}$
}

Fecha de recepción: 28-03-2018 - Fecha de aceptación: 04-07-2018

Hábitat y Sociedad (ISSN 2173-125X), n. ${ }^{\circ} 11$, noviembre de 2018, pp. 223-238.

http://dx.doi.org/10.12795/HabitatySociedad.2018.i11.13

\begin{abstract}
The history of women architects has not been told in the same way as that of their male colleagues. At this point, the actions of invisibility that were carried out to suppress female architects of history become more than evident.

Un día | una arquitecta is a collective that rescues the stories of women architects ignored by history, in their different areas of action. Our website publish online the results of this research, it makes available a biography of a women architect per day (Collective Un día | una arquitecta, s.f.).

During 2016, the project "Architects on the web" was carried out, in collaboration with the International Archive of Women in Architecture -IAWA - located at Virginia Tech, United States. This work won the 2016 Milka Bliznakov Prize, for its contribution to research and the dissemination on the web of the contributions of women in architecture.
\end{abstract}

\section{Key words}

Gender; Women Architects; Web Publication; International Archive of Women in Architecture

\section{Resumen}

La historia de las arquitectas no ha sido contada de igual manera que la de sus colegas varones. A este punto se hacen más que evidentes las acciones de invisibilización que se han seguido para suprimir a las arquitectas de la historia.

Un día | una arquitecta es un colectivo que rescata las historias de las arquitectas ignoradas por la historia, en sus diferentes áreas de actuación. Como producto de la investigación deriva la publicación en un sitio web que pone a disposición una biografía de una arquitecta por día (Colectivo Un Día | Una Arquitecta, s.f.).

Durante 2016 se llevó adelante el proyecto "Arquitectas en la red", junto con el Archivo Internacional de Mujeres en Arquitectura -IAWA- localizado en la Universidad Virginia Tech de Estados Unidos. Este trabajo fue ganador del Premio Milka Bliznakov 2016, por su contribución a la investigación y por la difusión en la web de las contribuciones de las mujeres en la arquitectura.

\section{Pallabras clave}

Género; Arquitectas; Publicación Web; Archivo Internacional de Mujeres en Arquitectura

\footnotetext{
1 Arquitecta; docente en la Facultad de Arquitectura Urbanismo y Diseño de la Universidad Nacional de Córdoba (Argentina); editora y redactora del blog “Un día/una arquitecta”. E-mail: arqmarciani@gmail.com.

2 Doctora en Arquitectura; investigadora del Conicet (Consejo Nacional de Investigaciones Científicas y Tecnológica); directora de la Maestría en Diseño de Procesos Innovativos en la Universidad Católica de Córdoba (Argentina), coordinadora de "Un día | una arquitecta”. Hidalgo 1540, CABA, Argentina. E-mail: ines.moisset@gmail.com.
} 


\section{Introducción}

La historia de las arquitectas no ha sido contada de igual manera que la de sus colegas varones. Las mujeres han sido borradas como objeto y sujeto de conocimiento, y la Historia solo ha reconstruido los registros masculinos de clases dominantes occidentales, y ha borrado, entre otras trayectorias, las femeninas.

Señala María Elena Díez Jorge que, a pesar de los obstáculos, las dificultades y las prohibiciones del sistema patriarcal en los distintos períodos históricos, "ello no implica pensar que las mujeres fueran únicamente agentes pasivos de la historia y víctimas de un sistema opresor. Las mujeres participaron y participan activamente en la historia y por ende en la historia de la arquitectura" (Diez Jorge, 2014, p. 182).

A partir de nuestras investigaciones hemos determinado que la presencia de las mujeres en los libros de historia de la arquitectura es muy escasa. Si buscamos en la biblioteca algunos de los diccionarios más reconocidos encontramos que sólo el $1 \%$ de las voces se refieren a arquitectas o estudios donde han participado arquitectas. El 99\% restante es dedicado a arquitectos o estudios conformados solamente por varones.

\section{Modern Architecture}

ED. Peter Gössel, Taschen 2007
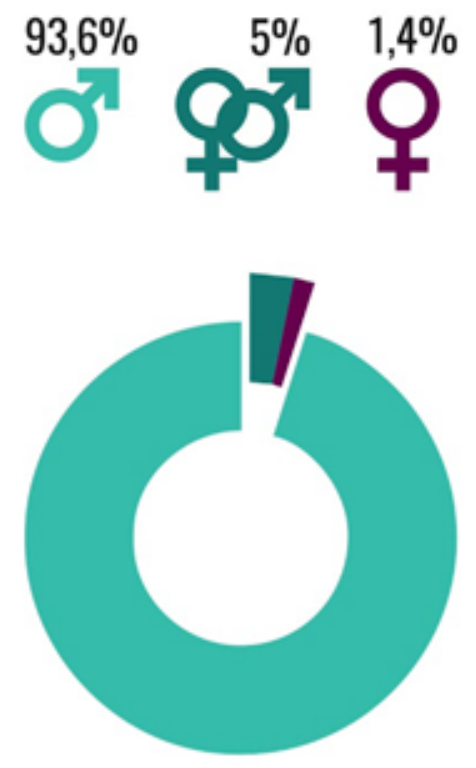

Figura 1: Gendercheck de las publicaciones Modern Architecture, Taschen (2007), Enciclopedia de la Arquitecura del siglo XX, Gustavo Gili (1989) y Diccionario de Arquitectura en Argentina de Clarín (2004). Fuente: Florencia Marciani, enero 2017

\section{Enciclopedia de la} arquitectura del siglo XX

Ed. Lampugnani, Gustavo Gili. 1989.
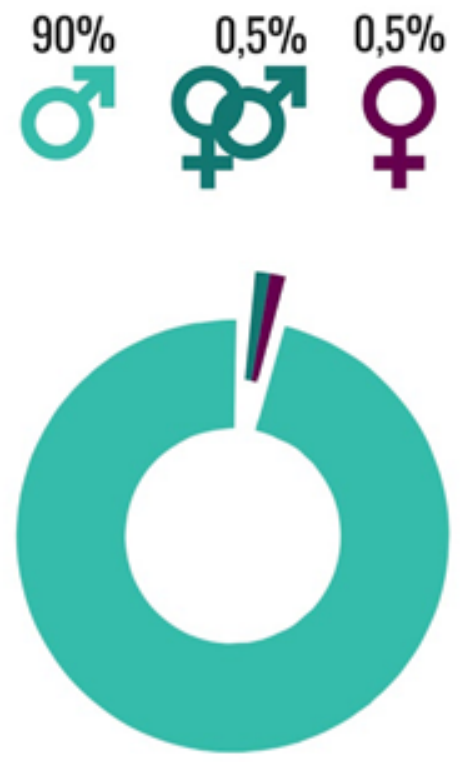

\section{Diccionario de Arquitectura} en Argentina

Clarín. 2004.
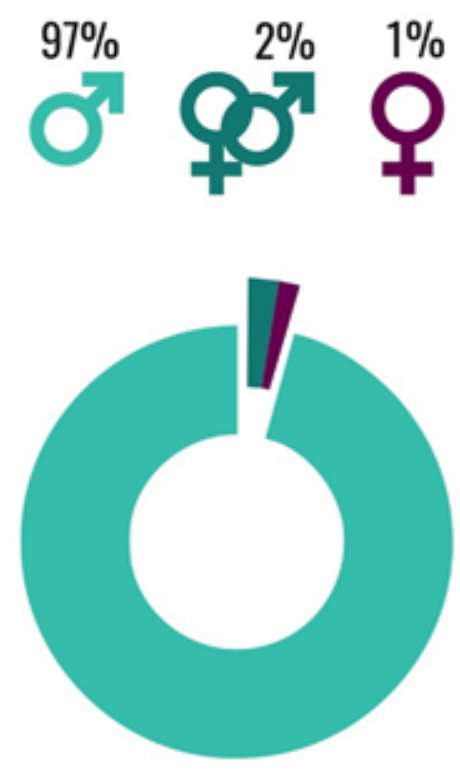

A este punto se hacen más que evidentes las acciones de invisibilización que se han seguido para suprimir a las arquitectas de la historia. Joanna Russ (1983), en su libro How to suppress womens writing identifica once métodos comunes que suelen ser usados para ignorar, condenar o minimizar el trabajo de las autoras. En la historia de la arquitectura encontramos procedimientos similares:

- Negación de la autoría, como por ejemplo obras asignadas a los esposos, hermanos o socios de las mujeres, a veces llevada a cabo por los historiadores como un sesgo inconsciente, como el caso de Plautilla Bricci (Muxi, 2015). 
- Mala fe, como en el caso de Louis Kahn que presentó en el MoMA el rascacielos de Filadelfia que diseñó Anne Tyng (Moisset, 2015) o Patrick Schumacher diciendo que es más autor él que Zaha Hadid en una entrevista (Alvarez y Gomez, 2017).

- Impedimento de firmar las obras, a veces por normas legales y a veces por casos como el de Nikolaus Pevsner que no permitía que Sadie Speigh firmara los artículos en Architectural Review (Ojeda, 2015).

- Prohibición del acceso a la formación, como en el caso de Gropius que consideraba que las mujeres no debían estudiar arquitectura en la Bauhaus, aun en contra de las leyes de la República de Weimar que establecían la educación universal (Gropius, 1956).

- Falsa categorización, es decir pretender que una arquitecta sea la clienta, la musa, la amante o la esposa del arquitecto. Tal es el caso de Truus Schröder, socia de Gerrit Rietveld (Moisset, 2017).

- Minimización de los aportes, como cuando Le Corbusier señala que Charlotte Perriand borda almohadones (Marciani, 2015).

- Reforzamiento de estereotipos, al subrayar que la arquitectura no es un campo para las mujeres como lo hace Carlo Scarpa (Bione, 2005).

Reconocer que las mujeres tienen una historia propia supone un cambio a nivel global, una nueva forma de mirar e interrogar a los documentos. Esto también significa replantear qué significa la disciplina, habitualmente centrada en el héroe o el genio, el arquitecto blanco que se dedica a proyectar y construir edificios.

\section{Un día | una arquitecta}

En los últimos años se han incrementado los estudios que ponen en evidencia los sesgos de género en las profesiones relacionadas al diseño. Desde el equipo de Un Día | Una Arquitecta trabajamos desde 2015 para poner a disposición diaria en las redes sociales y hacer visible en idioma español, el aporte de las arquitectas en diferentes facetas. De este modo, se trata de proponer una mirada crítica a la escritura de la historia desde puntos de vista exclusivamente masculinos.

Consideramos el uso de las redes sociales como instrumentos de empoderamiento político. Para promover el equilibrio creemos que es necesaria una revisión de la historia. Las biografías sirvieron en principio para ir detectando las protagonistas y organizar la información que no se incluía en libros de historia de la arquitectura. Iniciamos pensando en un proyecto de una duración de un año donde publicaríamos diariamente en nuestro sitio web una biografía de una arquitecta por día. El sitio ha superado el millón de visitas.

El proyecto se extendió publicando unos 800 artículos y en estos momentos estamos planificando una cuarta temporada. Además, se fueron ramificando acciones que tenían que ver con la promoción de las mujeres en la profesión. El sitio con las biografías sirvió como plataforma que se extendió a otros espacios. La página de Facebook tiene más de 20000 seguidores. También estamos presentes en Twitter y en YouTube.

Las tareas llevadas adelante por el equipo implican no solo el trabajo de redacción, sino también de recopilación de información ya 
que, aunque existe material publicado de muchas de las arquitectas que presentamos, gran parte está disperso y en otros idiomas.

Acceder a los espacios donde se construye la opinión pública es una manera de generar reflexión y concienciar para que los reclamos y la necesidad de reivindicación provengan desde la sociedad (botton-up). El activismo feminista es un tipo de activismo que trabaja para sensibilizar, proteger e impulsar los derechos del colectivo femenino.

Por esta labor obtuvimos en 2016 el Premio Milka Bliznakov del International Archive of Women in Architecture que tiene sede en la universidad de Virginia Tech.

\section{Archivo Internacional de Mujeres en la Arquitectura}

A lo largo de la historia el trabajo de las mujeres ha sido infravalorado por lo que existe dificultad para encontrar fuentes documentales. Por ejemplo, debido a obstáculos legales, las mujeres no podían firmar las obras, como tampoco contratos. Los archivos, en general, reflejan y reproducen estas prácticas y tensiones sociales que moldean la manera en que se escribe la historia. Al mismo tiempo sirve para legitimar y otorgar credibilidad a lecturas y narrativas Aguirre Ramirez y VillaFlores, 2009). Para Eric Ketelaar (2007) los documentos y los archivos pueden ser instrumentos de poder, pero, paradójicamente, los mismos documentos pueden convertirse en instrumentos de empoderamiento y liberación, salvación y libertad.

El trabajo realizado por el Archivo Internacional de Mujeres en Arquitectura (IAWA) es una acción para generar un cambio en esta situación de desigualdad y representa una gran fuente de información sobre arquitectas que no estaban al alcance público.

Fue fundado en 1985 por la arquitecta Milka Bliznakov con el propósito de documentar la historia de las contribuciones de las mujeres al medio ambiente construido. Después de más de una década de enseñanza y estudio de la arquitectura, Bliznakov, inspirada en el buen desempeño de algunas de sus estudiantes mujeres que cuestionaron por qué en cinco años de estudio no habían conocido ni estudiado el trabajo de arquitectas mujeres, identificó la escasa documentación, los grandes vacíos y contradicciones sobre el desempeño profesional de las mujeres en este ámbito. Esta estremecedora revelación, combinada con la frustración por la falta de recursos y fuentes confiables, la volcaron a centrar sus estudios en el papel de las mujeres arquitectas en la historia (Rivera, 2015).

El trabajo de Milka Bliznakov comenzó en 1983 cuando inició acciones para corregir la omisión de las mujeres de la historia de la arquitectura, asegurando que las generaciones futuras no pueden decir, simplemente por falta de información, que las arquitectas nunca hicieron nada (Zellner, 2016). Escribió más de 1000 cartas a arquitectas de todo el mundo, con la esperanza de recibir material para preservarlo (ob. cit.).

El IAWA trabaja recolectando, preservando y proporcionando acceso a los registros de las organizaciones arquitectónicas de mujeres y los documentos profesionales de mujeres arquitectas, paisajistas, diseñadoras, urbanistas, historiadoras y críticas.

En los primeros años recopilaron documentos de las mujeres pioneras que practicaron la profesión en un momento en el que había pocas mujeres en el campo. Sin embargo, actualmente documenta todas las 
generaciones de mujeres en la arquitectura con el fin de reducir la brecha en la disponibilidad de material de origen primario para la investigación arquitectónica, de la mujer y de la historia social.

Actualmente el archivo dispone de 450 colecciones y las mujeres representadas en las colecciones vivieron, enseñaron y trabajaron en más de cuarenta países de los cinco continentes.

El archivo se transforma en un elemento estratégico para la práctica de la historia y la memoria justa.

\section{Arquitectas en la red}

Durante 2016 Un día | una arquitecta llevó adelante el proyecto "Arquitectas en la red", ganador del premio a la Investigación Milka Bliznakov 2016 por su contribución a la investigación y por la difusión en la web de las contribuciones de las mujeres en la arquitectura. Este premio, entregado anualmente, fue compartido en esta ocasión con el trabajo de la Dra. Tanja Poppelreuter, de la Universidad de Ulster en Belfast, con su propuesta, "Refugee and émigré female architects before 1940".

El proyecto fue desarrollado por un grupo de redactoras argentinas (Silvina Barraud, Cecilia Kesman, Florencia Marciani, Beatriz Ojeda y Marcela Roitman) y estuvo coordinado por Inés Moisset.

Los objetivos principales del proyecto fueron, por un lado, visibilizar el trabajo de mujeres que trabajaron en el campo de la arquitectura en todo el mundo y por otro, difundir material que no se encontraba disponible.

Se trabajó de manera sinérgica junto al Archivo Internacional de Mujeres en Arquitectura (IAWA), tanto de manera presencial como a distancia. La metodología se basó en la búsqueda bibliográfica exhaustiva a partir de una selección de casos. Considerando la disparidad de contenido entre las colecciones del IAWA, se tuvieron en cuenta algunos criterios a la hora de seleccionar las biografías. Siguiendo con las lineas generales del blog, se propuso reseñar arquitectas que hubieran estudiado formalmente. Además, se verificó que cada colección contara con cantidad y diversidad de material suficiente. Fue imprescindible que contuvieran textos e imágenes.

El trabajo se desarrolló en cuatro etapas:

- revisión y selección de las colecciones;

- recopilación de la información;

- traducción del material y redacción de cada biografía;

- publicación en las redes.

En primera instancia se trabajó con la guía de colecciones y con el listado de contenidos de cada una de ellas disponibles online (Guide to the IAWA Collections, s.f.). Del listado general se seleccionaron 50 colecciones de mujeres arquitectas relevantes de todo el mundo, las cuales se detallan en las Figuras 2, 3, 4, 5 y 6. 


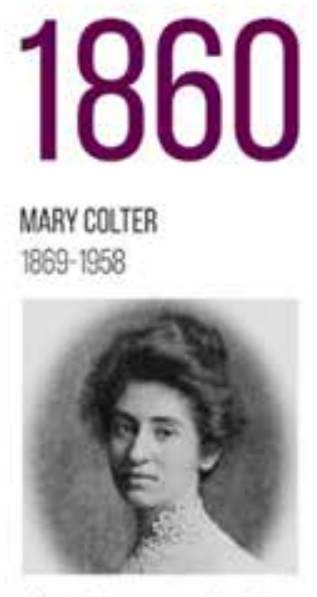

Arquitecta estadouni dense que diseñó para la empresa Fred Harvey a principios del siglo $\mathrm{XX}$ y definió un estilo que fusionó tradición neocolonial y americana.

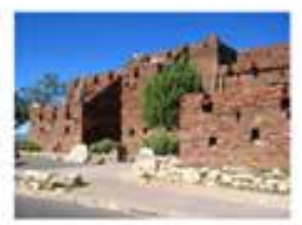

Mary Colter. Hopi Housc, 1904

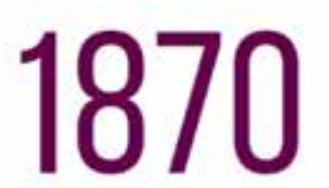

MARY ROCXWELL HOOK 1877-1978

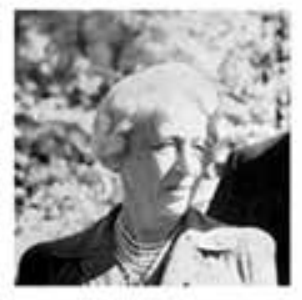

Emblemática arquitec ta de Kansas, distingui da por el diseño de viviendas con carácter $y$ sello personal.

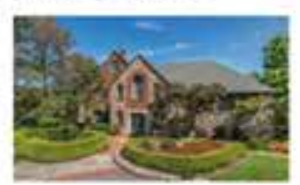

Mary Rockwell Hook. Casa Rockwell.

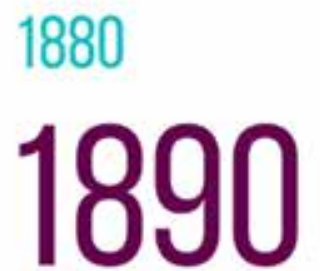

HENRIETTA MAY STENMESCH

$1893-1979$

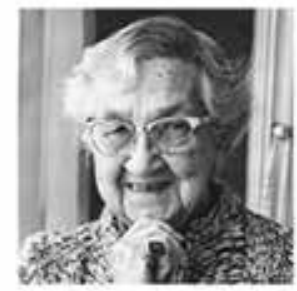

Fuc una de las

fundadoras de la

Cofradía Alongine,

germen de la

Asociación de Mujeres

en Arquitectura.

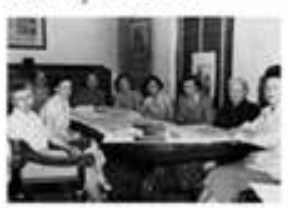

H. May Steinmesch.

Reunión de AWA. 1950.

\section{ALEXANDRA BERIUKOVA}

1895-1967

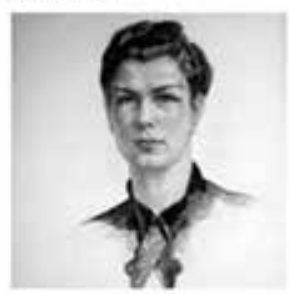

Arquitecta rusa

pionera en la introduc ción de la arquitectura moderna en Canadá.

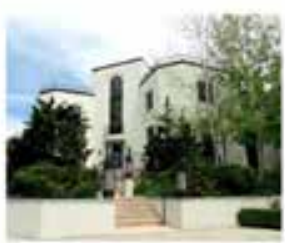

Alexandin Biriukova. Casa Lawren Harris.

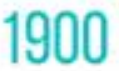

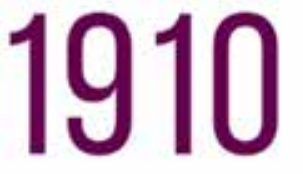

HELENE KOULR-BUCHWIESER 1912-2008

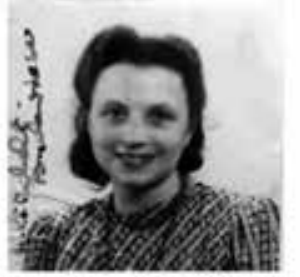

Arquitecta $\mathrm{e}$ ingeniera civil austriaca. Trabajó activamente en la reconstrucción de Viena luego de la Scgunda Guerra

Mundial.

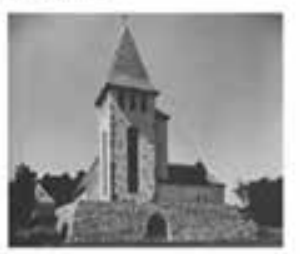

Helene Koller-

Buchwieser, Iglesia

para servicios

Protestantes y

Católicos.

\section{LISBETH SACHS}

1914-2002

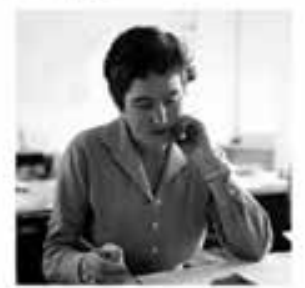

Arquitecta, ingeniera y crítica suiza.

Desarrolló numerosos proyectos en Zúrich y trabajó en la obra de la

Villa Mairea.

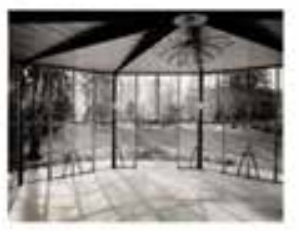

HAN SCHROODER

1918-1992

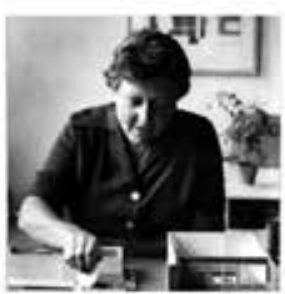

Fue la primera

graduada que practicó

la arquitectura en

Holanda. Vivió en la casa Schröder, junto a su madre, $y$ fue colaboradora de

Rietveld.

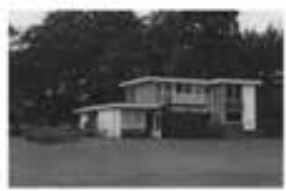

Han Schröder, casa para su hermano B.F. Schröder en Hattem, 1957.

Lisbeth Sachs. Foyer de Kurtheaters Baden
ZELMA WILSON

$1918 \cdot 1996$

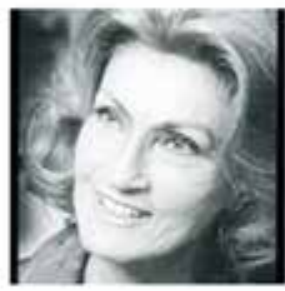

Fue la primera graduada que practicó la arquitectura en Holanda. Vivió en la casa Schröder, junto a su madre, $y$ fue colaboradora de Rictveld.

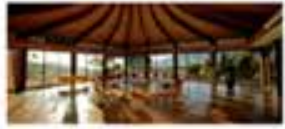

Zelma Wilson,

Meditation Mount, Ojai.

JANEC. HALL JOHNSON 1919-2001

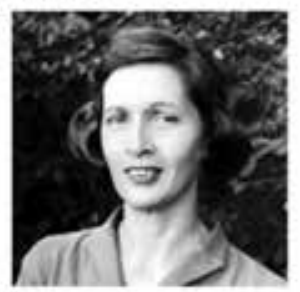

Jane C. Hall, ingeniera $y$ arquitecta norteame ricana. Diseñó

numerosos emprendi mientos comerciales, residenciales $\mathrm{C}$

institucionales en St. Louis.

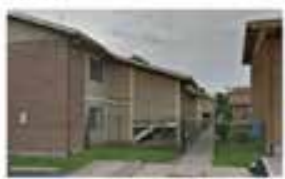

Jane C. Hall Johnson. Villa de Retiro, St. Louis 1980.

Figura 2: Líneas de tiempo que organizan las arquitectas según el año de nacimiento, parte 1. Fuente: Florencia Marciani, enero 2017. 


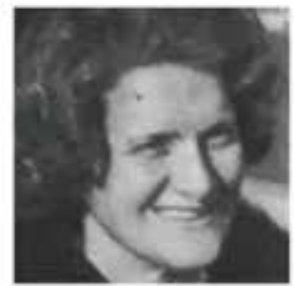

Arquitceta austriaca. Trabajó en diseño de edificios, en planifica ción urbana, en restauración y en docencia.

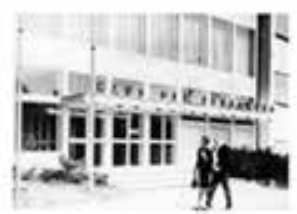

Hlse Koci, Casa de encuentro, 1969.

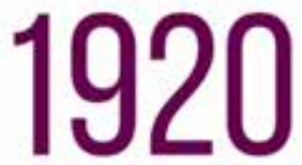

HANNA ADANCZEWSKAWEJCHERT

1920-1996

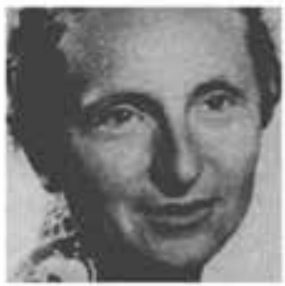

Hanna Adamezewska Wejchert fue arquitecta y urbanista polaca. Autora de numerosos planes de desarrollo urbano.

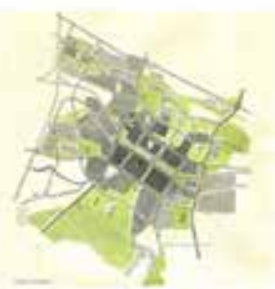

Hanna AdamczewskaWejchert. Plan urban de la ciudad de Nueva Tychy.
EVAKRUGEER

1922

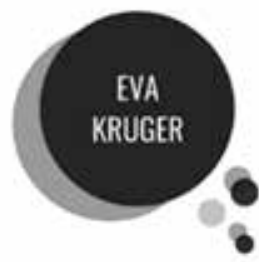

Arquitecta alemana, participó en el diseño de interiores de varios salones del Palacio de Bellevue.

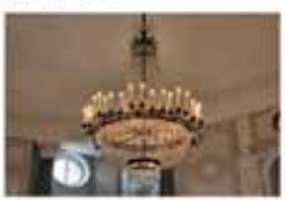

Eva Kruger. Candelabros Palacio de Bellevue

JEAN LNDEN YOUNG 1922-1997

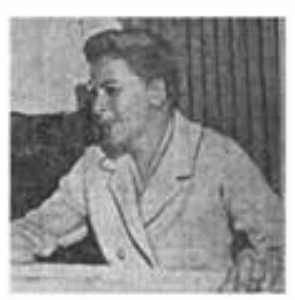

Arquitecta estadouni dense, trabajô activamente para mejorar las condicio nes labomles de las mujeres en la profe sión.

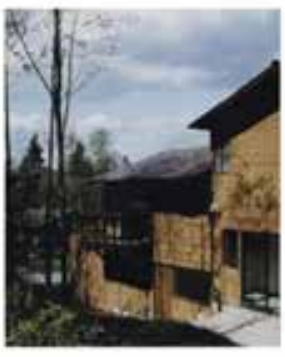

Jean Young Vivienda Yarrow:

\section{TSVETANA NENOVA 1923-2015}

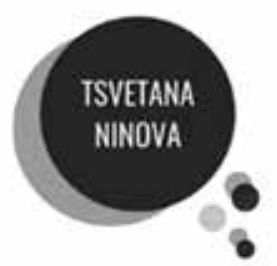

Arquirecta y urbanista de Bulgaria, desarrolló grandes proyectos urbanos y edificios gubernamentales en todo cl pais.

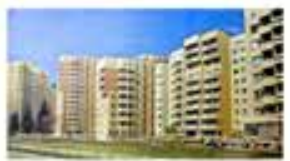

Tsvetana Ninova.

Edificio de vivienda colcetiva Beli Brezi, Sofia, Bulgaria, 1977 1989.
DNA STANCHEVA

1925

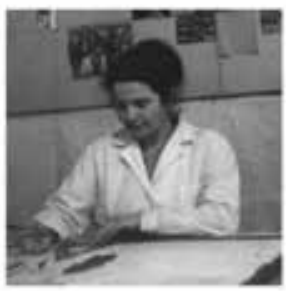

Arquitccta búlgara, ha recibido premios por sus destacadas contribuciones a la arquitectura.

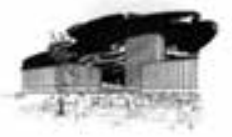

Dina Stancheva,

Concurso para la Casa de los Soviets, 1958.

\section{LUOXIAOWE}

1925

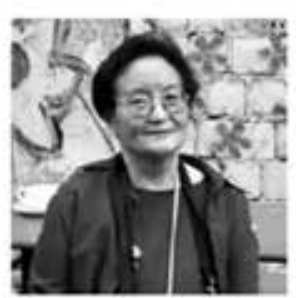

Presidenta de la

Sociedad de

Arquitectura de

Shanghai $y$ profesora visitante en instituciones de nivel internacio. nal.

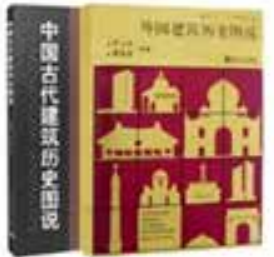

Luo Xiaowei, Una historia ilustrada de la arquitectura occidental, Tongii University Press.

1986.

(n)

MARTHA L CRAWFORO 1925-1994

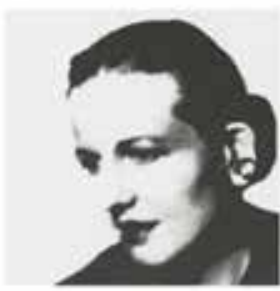

Diseriadora de interiores, una de las principales influencias en el campo del discno comercial en Estados Unidos de los años 60

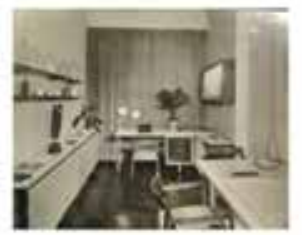

Martha Crawford, Discño de interiores.

MARJORIE MEAD HOOKER 1925.2006

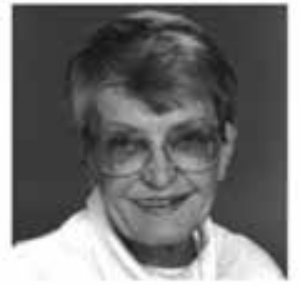

Arquitecta de Estados Unidos. Es pionera en varios aspectos: una de las primeras graduadas de su universidad, primera mujer en afiliarse y presidir diversas instituciones en Nuevo México.

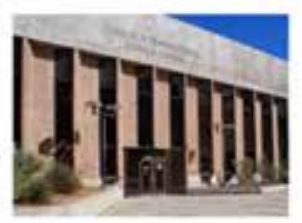

Peggy Hooker, con Flatow, Moore, Bryan y Fairburn, Cancer Research Center, Universidad de Nuevo Méxica.

Figura 3: Líneas de tiempo que organizan las arquitectas según el año de nacimiento, parte 2. Fuente: Florencia Marciani, enero 2017 


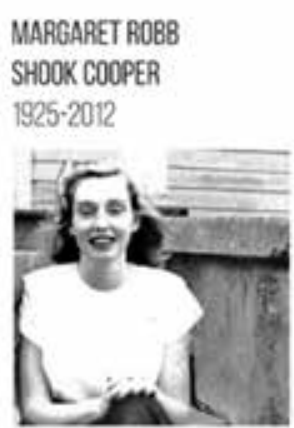

Arquitecta norteameri cana activa organizado $\mathrm{ra}$ de Women in Architecture en Washington DC.

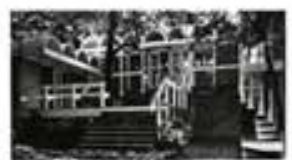

Margaret Robb Shook Cooper, Casa en Pine Knoll Shores, 1975.

ANNA CAMPBELL BLISS $1925-2015$

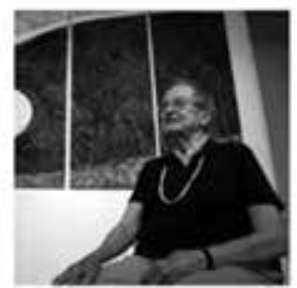

Anna Campbell fue arquitecta y artista, pionera en la explon ción entre color, matemáticas y sistemas de computación para la generación de arte.

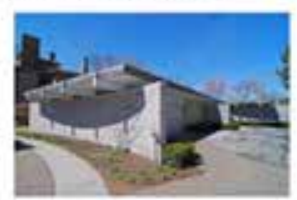

Anna Campbell Bliss. Bliss \& Campbell. Vivienda Dalyrympl, Minneapolis

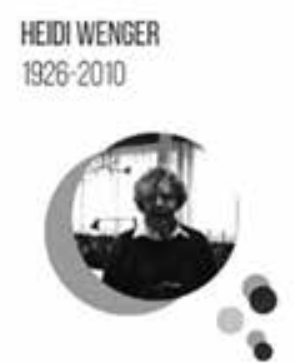

Arquitecta suiza que lleva adelante un método de indągación entre lo existencial y lo técnico en la creación arquitectónica.

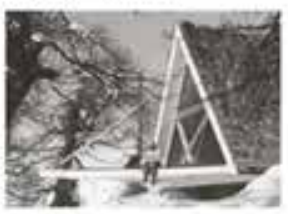

Heidi y Peter Wenger, Casa Trigon, Saflisch.

\section{WENA WALDNER DOWS} 1928

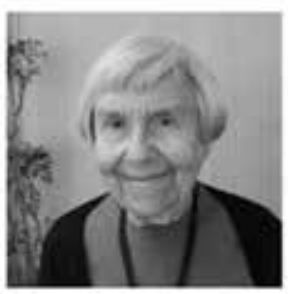

Arquitecta norteameri cana, se especializa en la remodelación de viviendas en California, donde trabaja entre 1952 y 2014.

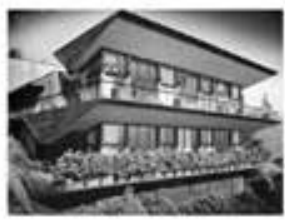

Wena Waldiner Dows, Casa Harwell

Hamilton Harris.

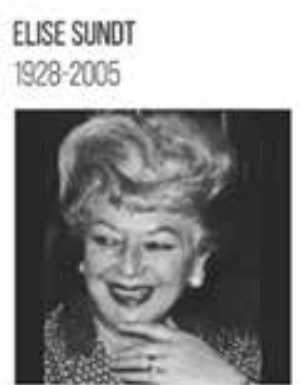

Arquitecta austriaca. Fue pionera en sistemas y procedi mientos de construc ción prefabricada de su pais.

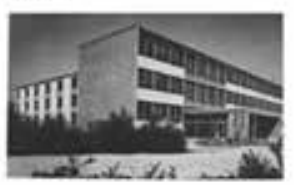

Elise Sundt, Colcgio en calle Roda Roda Gasse 3. Viena.

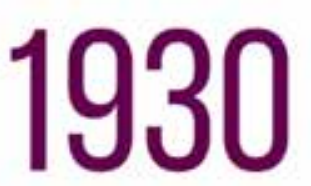

MASAKO HAYASH 1928-2001 HATSUE YAMADA 1930 NOBUKO NAKAHARA 1929-2008

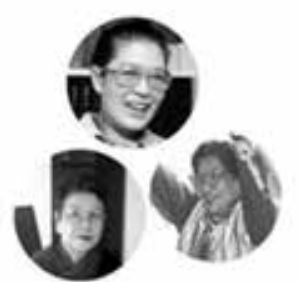

Pioneras japonesas que establecicron la firma Hayashi, Yamada y Nakahara, Círculo de Diseño Arquítectónico en los 50 .

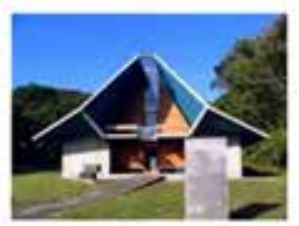

Masako Hayashi,

Hayashi, Masada,

Nakaraha, Circulo de diserio arquitectónico, Seashell Gallery, 1967

\section{DIANA BALMORI}

$1982 \cdot 2016$

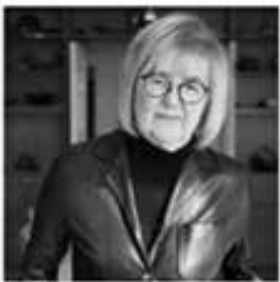

Arquitecta dedicada al disen̂o urbano y del paisaje es titular de Balmori Associates desde 1990 .

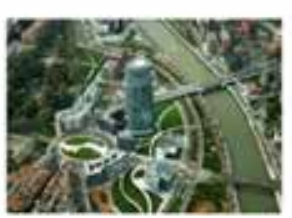

Diana Balmori, Master Plan de Bilbao.

\section{ELSALEVISEUR}

1932

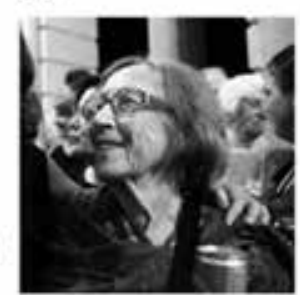

Arquitecta sudafricana que trabajó en

Inglaterra y Estados Unidos especializada en paisaje y ecologia.

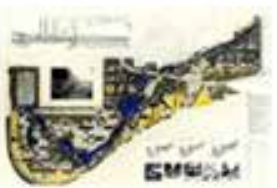

Elsa Leviseur, UC Davis Arboretum

Figura 4: Líneas de tiempo que organizan las arquitectas según el año de nacimiento, parte 3. Fuente: Florencia Marciani, enero 2017.

\section{EUSABETH STEINEGGER \\ 1933-2008}

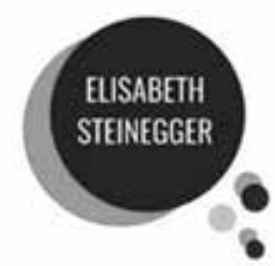

Arquitecta suiza, socia de Steinegger + Hartmann, estudio especializado en establecimientos educativos y centros de rehabilitación.

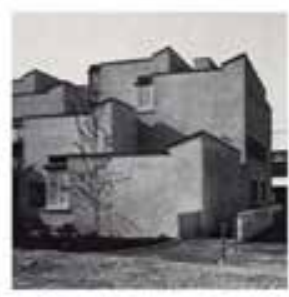

Elisabeth y Jcan Claude Stcinegger,

Escuela Sccundaria en Sissach.

\section{DOROTHEE STELZER KING 1934}

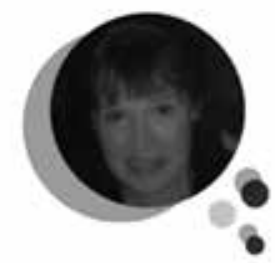

Arquitecta alemana. Trabajó en Alemania, Estados Unidos y Bahamas Se dedicó principalmente a obras de carácter social y a la docencia.

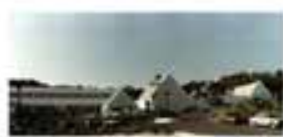

Dorothee Stelzer

King, Escuela de

Enfermeria de Nassau. 
MÁRIA DOMBAI

1934

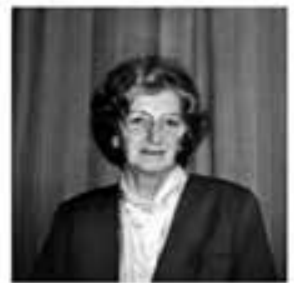

Arquitecta húngara especialista en construcciones para la industria alimenticia.

Ha realizado trabajos alrededor del mundo.

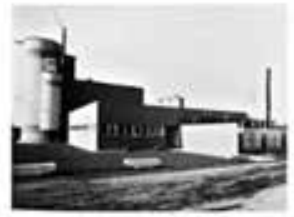

Margaret Robb Shook Cooper, Casa en Pine Knoll Shores, 1975

ROSARIA PIOMELLI

1937

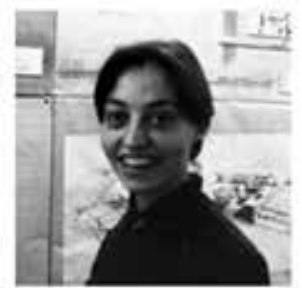

Gran educadora. Decana del City College de la "NYSA", fue la primera mujer Decana de una facultad de arquitectu ra en los Estados Unidos

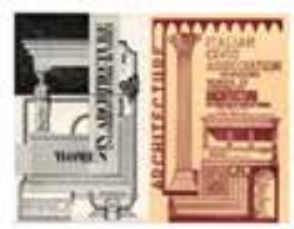

Rosaria Piomelli,

Muestra Mujeres en arquitectura
HELGA SCHMDDT-THOMSEN 1938

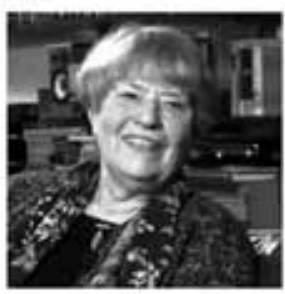

Gran arquitecta alemana, realizó numerosas obras, escribió sobre arquitectura y sobre el rol de las mujeres en la profesión.

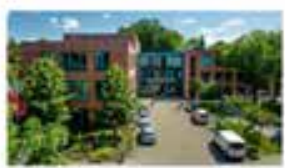

Helga Schmidt

Thomsen, complejo de radio "ORB PotsdamBabelsberg"

UTE WESTRÖM

1939

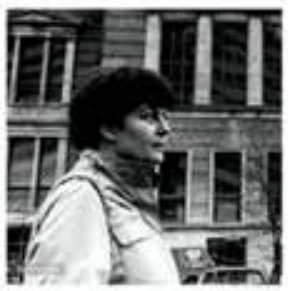

Arquitecta alemana que reconstruyó el "Martin Gropius Bau" en Berlin, un centro internacional de arte.

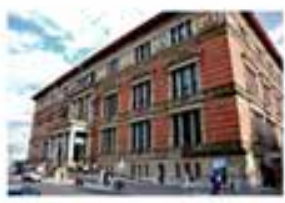

Ute Westrồm y

Winnetou Kampmann, reconstrucción MartinGropius-Bau.

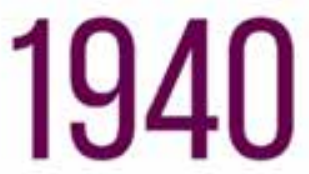

VERENA DIETRICH

$1941-2004$

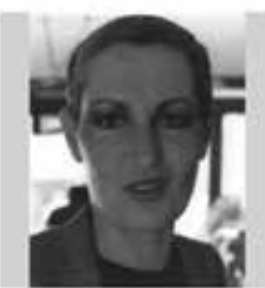

Arquitecta y profesora universitaria alemana.

Fue una de las arquitectas más reconocidas de Alemania.

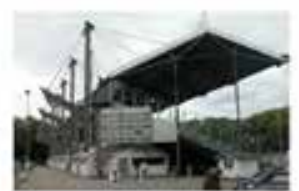

Verena Dietrich,

Tribuna Sportpark

Höhenberg

GHSLAINE HERMANUZ 1942

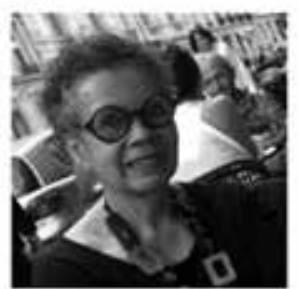

Arcquitecta y urbanista suiza radicada en Nueva York. Trabaja para el desarrollo urbano de los más necesitados.

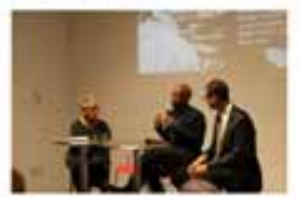

Ghislainc Hermanuz, Comité de la diversidad y la inclusión AIANY

Diversity y nycobaNOMA.
INGEBORG KUHLER

1943

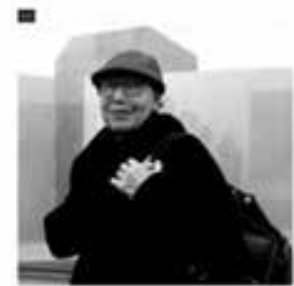

Arquitecta alemana, profesional indepen diente, docente, miembro y presidenta de junta consultiva de disçño de Salzburgo

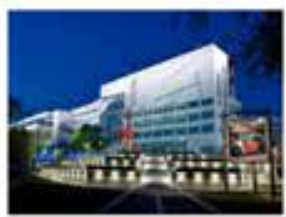

Ingeborg Kuhler, Technoseum

KERSTIN DÓRHŎFER 1943

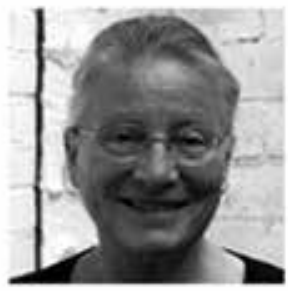

Profesora y Doctora en Arquitecrura y Urbanismo alemana. Su investigación se centra en los estudios urbanos y las cuestio nes de género.

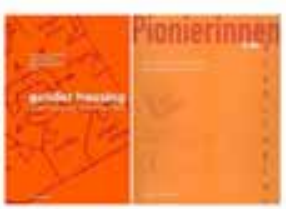

Kerstin Dörhöfer, Libros: Gender housing $y$ Pionerinnen in der Architektur.

\section{MAIJA HAKALA-MEYER}

1944

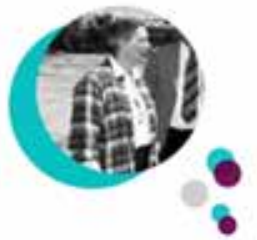

Se dedica a la arquitec tura en Alemania, proyectando $y$ construyendo importantes edificios.

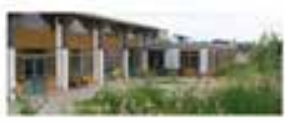

Maija Hakala y Dirk Meyer, Werkstäten der Lebenshilfe,

Braunschweig, 2001

Figura 5: Líneas de tiempo que organizan las arquitectas según el año de nacimien-

to, parte 4. Fuente: Florencia Marciani, enero 2017 
ALDA SANTOS

1945

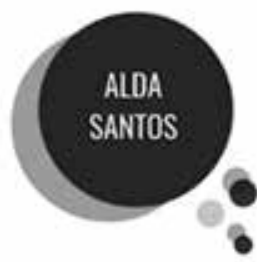

Arquitecta de origen portugués, que aporta a la disciplina, articulando diversos procesos transcultura les

\section{CRISTINA GRAU}

1946. 1997

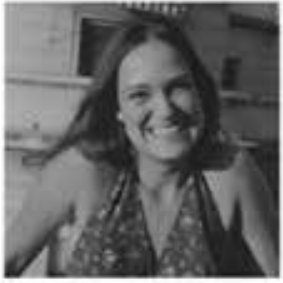

Arquitecta y profesona española, autora del libro Borges y la arquitectura.

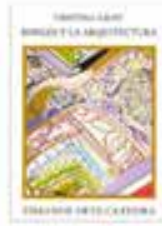

Cristina Grau, Libro: Borges $y$ la arquitectura.

\section{DIANE LEGGE KEMP 1949}

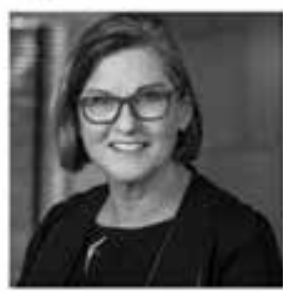

Arquitecta estadouni dense que cuenta con décadas de experiencia en proyectos de paisaje y urbanisma

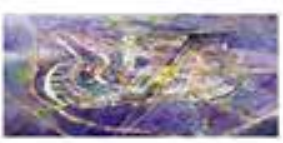

Diane Legge Kemp. CallisonRTKI.

Estación de trencs Guangehou.

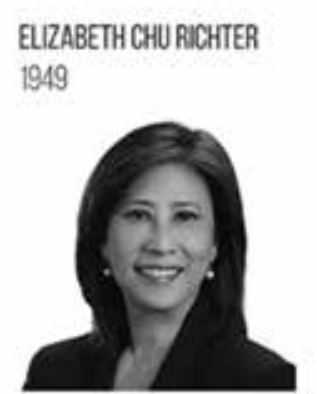

Arquitecta,

Vicepresidenta de Sociedad de Arquitectos, Texas y Presidenta del Instituto Americano de arquitectos.

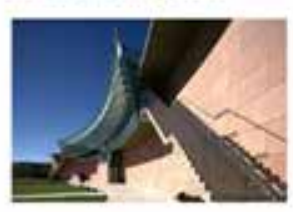

Elizabeth Chu Ritcher, Teatro DMC.
KRISTINE K.FALLON

1949

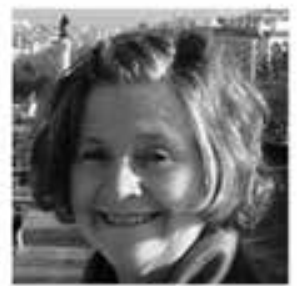

Arquitecta estadouni dense pionera en la aplicación de tecnolo gias de la información a la arquitectura.

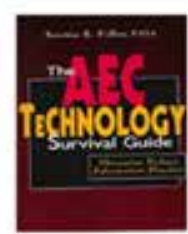

Kristine K. Fallon. Libro The AEC technology survival guide. John Wiley \& Sons. 1997.

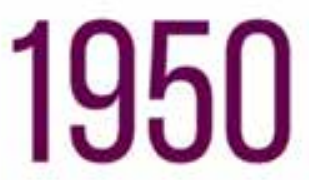

LOUISE ST. JOHN KENNEDY 1950

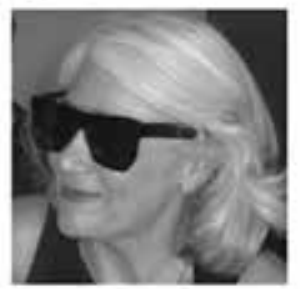

Arquitecta australiana con múltiples premios por su labor, que tiene formación en psicología.

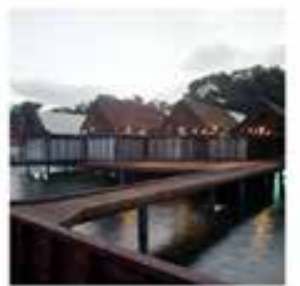

Louise St John

Kennedy. Casa de Te

Mosman bay,1986.

\section{MARIA AUBOČCK}

1951

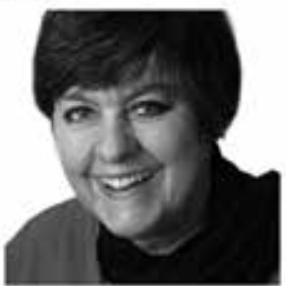

Arquitecta paisajista austritica fundadora de Auböck + Kárász y presidenta del prestigioso Taller Werkstätte Carl Auböck.

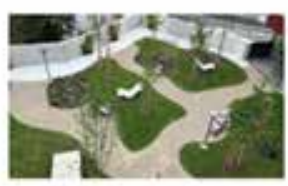

Maria Auböck.

Auböck + Kánísz.

Smart Housing Viena. 2016.

\section{DOINA MARILENA CIOCANEA} 1951

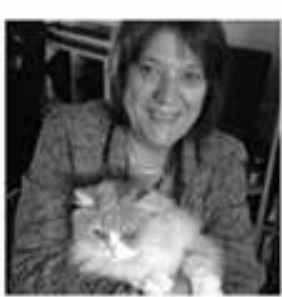

Arquitecta y urbanista rumana. Participó cn la construcción del Parlamento Rumano

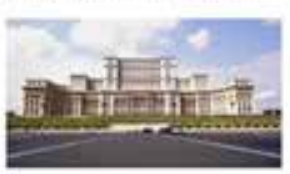

Doina Marilena

Ciocainea. Instituto de Diseño Carpati. Casa del Puebla

Parlamento de

Rumania. 1985-1990.
GEORGNALIPSEY

1953

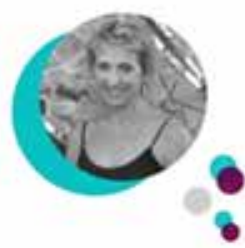

Arquitecta y artista plástica australiana, titular del cstudio Spatial Transformations con sede en Tucson, Arizona.

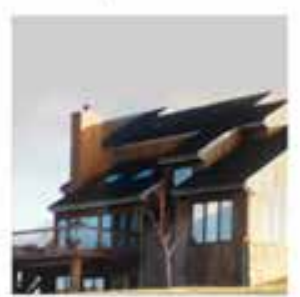

Geongina Lipsey, Herath, Robinson \& Mcl ean Custom

Residences

\section{SYLVIA FRITZ}

1955

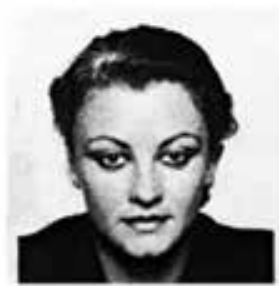

Arquitecta austriaca, nace y trabaja en Viena. Además, es pintora, escultora y diseñadora de interiores $\mathrm{y}$ de mobiliario

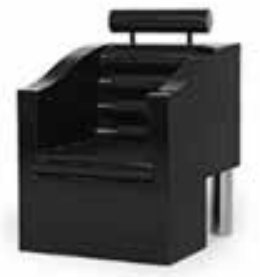

Sylvia Fritz, diseño de mobiliario para Missoni

Figura 6: Líneas de tiempo que organizan las arquitectas según el año de nacimiento, parte 5. Fuente: Florencia Marciani, enero 2017. 
Los perfiles seleccionados ilustran el trabajo que han desarrollado las mujeres desde principios del siglo $\mathrm{xx}$ hasta la actualidad. Y, como se puede verificar en las Figuras 7 y 8, también muestran cómo han llevado adelante la carrera profesional en diferentes países del mundo, principalmente de Estados Unidos, Alemania y de países de Europa del este, como Polonia, Hungría, Bulgaria y Rumania.
Figura 7: Distribución global según país de origen de las arquitectas en relación al total analizado. Relativos. Fuente: Florencia Marciani, enero 2017.
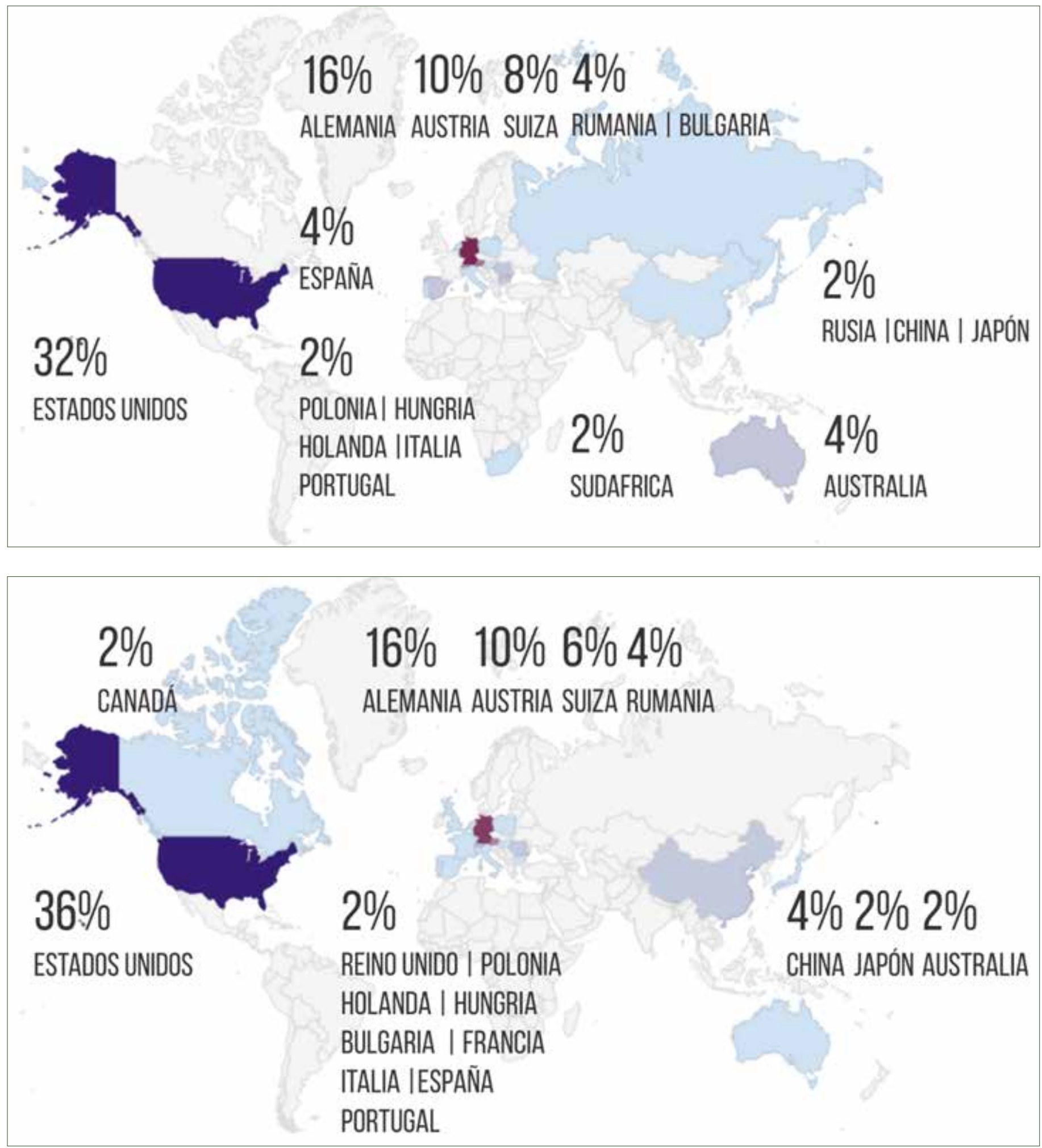

Figura 8: Distribución global según país de residencia de las arquitectas en relación al total analizado. Relativos. Fuente: Florencia Marciani, enero 2017. 
Figura 9: Número de arquitectas por área de trabajo. Absolutos. Fuente: Florencia Marciani, enero 2017.
En septiembre de 2016, una representante del equipo, Florencia Marciani, viajó a Virginia, Estados Unidos, para trabajar en la selección y recopilación del material disponible en el IAWA. Allí la recibieron Paola Zellner, secretaria del archivo, y Sam Win, archivista, con quienes trabajó arduamente durante el tiempo de desarrollo del proyecto.

Debido a la poca disponibilidad de tiempo, el trabajo en Virginia consistió principalmente en revisar y clasificar el contenido de cada colección y seleccionar el material necesario. El equipo del archivo fue el encargado de escanear y enviar el material vía email. Luego éste fue procesado por las redactoras en Argentina.

Los papeles personales, cartas, diarios personales, autobiografías, colecciones de fotografías, currículos, bitácoras de viaje, planos, documentos legales y contables, artículos de medios locales, entre otros documentos disponibles, revelan las diferentes facetas que las mujeres han podido desarrollar en la práctica de la arquitectura. Estas abarcan trabajos de gestión, de diseño y proyecto, trabajos a pie de obra, en la academia y en organizaciones de mujeres, entro otros (Figura 9). Cada elemento del archivo constituye una pieza que debe ser ensamblada y contextualizada para poder reconstruir las historias de las arquitectas. Las redactoras debieron construir a partir de las teselas documentales que se obtuvieron allí y sumar una búsqueda bibliográfica. Mucha documentación estaba disponible en idiomas extranjeros como chino, japonés, búlgaro, rumano, alemán e inglés, lo que significó un arduo trabajo de traducción.

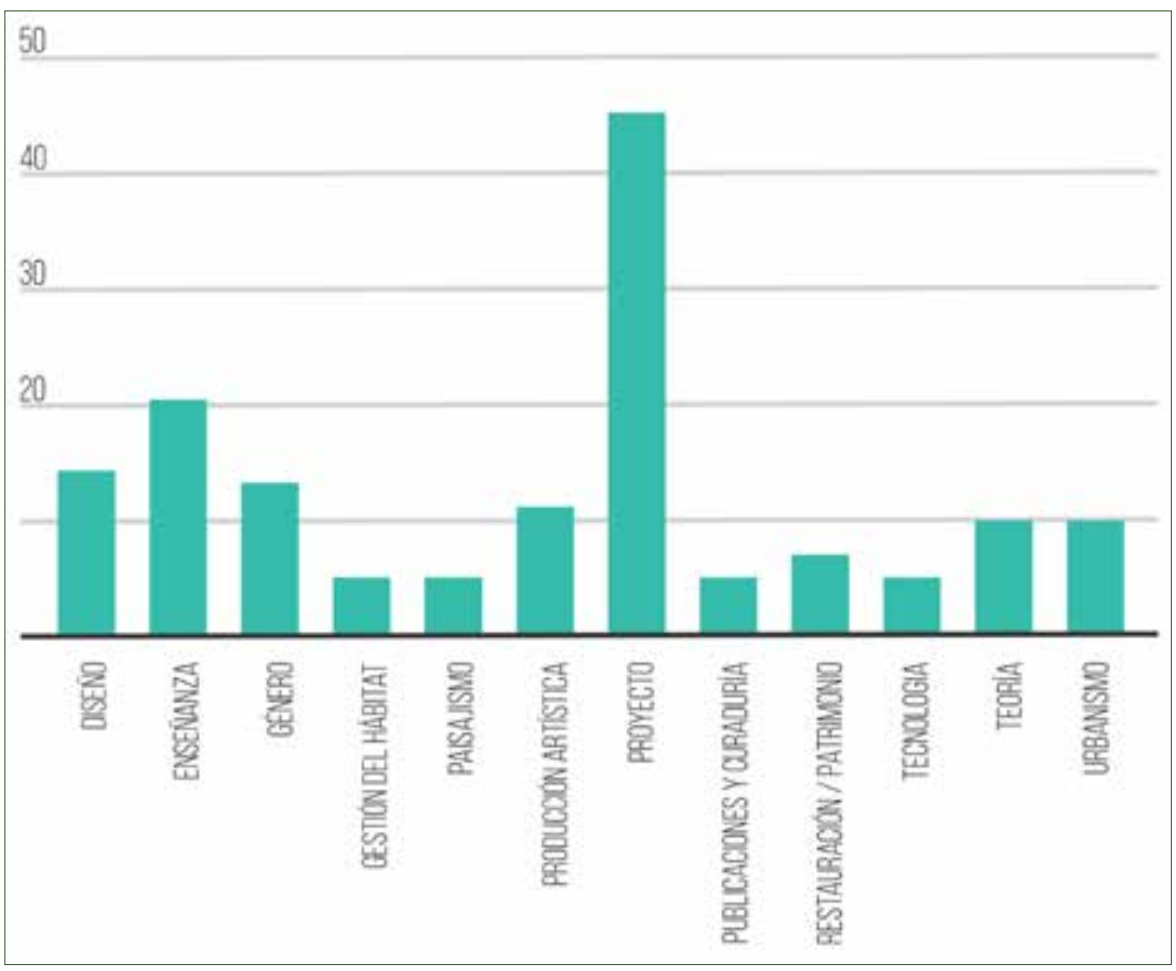

Entre las arquitectas reseñadas se encuentran figuras como Mary Colter, arquitecta estadounidense que trabajó en principios del siglo Xx. Entre sus trabajos destacados cabe especial mención su participación en el proyecto del Ferrocarril de Santa Fe y en el Parque Nacional del Gran Cañón del Colorado. Su labor definió así un estilo caracterizado por la conjunción de reminiscencias del neocolonial español con motivos nativos americanos; el mencionado estilo se convirtió en típico 
del suroeste de Estados Unidos, y es conocido actualmente como estilo Santa Fe (Barraud, 2016).

Otra biografía realizada fue la de Hanna Adamczewska Wejchert, una arquitecta y urbanista polaca, que trabajó en la época de posguerra en el Departamento de Planificación Urbana. Sus proyectos tomaron como referencia el modelo inglés de "new towns" y lograron introducir una expresión modernista en Polonia (Marciani, 2016).

El archivo permitió acceder a arquitectas del Lejano Oriente. Tal es el caso de Nobuko Nakahara que trabajó junto a sus socias Masako Hayashi y Hatsue Yamada, a partir de la década del 50 en Japón. Las arquitectas hicieron una reflexión crítica sobre los modos de vida de su país y cómo cambiaron después de la guerra, donde las casas tenían espacios de usos no determinados que son importantes para la mentalidad japonesa (Moisset, 2016).

También localizamos biografías de arquitectas con originales enfoques teóricos como Luo Xiaowei, arquitecta y académica de Shangai. Su investigación incluye también la historia de la arquitectura contemporánea china, especialmente el desarrollo de la disciplina y el rescate de su influencia a nivel internacional. Desde su producción teórica, Luo Xiaowei se ha opuesto fuertemente a la visión hegemónica occidental en el estudio de la historia de la arquitectura. Postula por primera vez en su país romper con el concepto eurocéntrico en la historia de la disciplina. En busca de ampliar su investigación toma referentes de la arquitectura islámica temprana y de oriente en general (Ojeda, 2016).

\section{Consideraciones finales}

Solo este pequeño muestreo basta para ver la diversidad de perfiles albergados en el Archivo. Como ha dicho Natalie Zemon Davies (1999), las biografías de las mujeres que ella denomina "de los márgenes" informan más de las sociedades en las que vivieron que muchas otras historias. Son la forma primigenia de la historia de las mujeres.

Cada biografía es una puerta que se abre para permitirnos descubrir el trabajo, los logros y las dificultades que enfrentaron estas profesionales. Son diferentes maneras de desarrollar la profesión donde podemos encontrar hoy, más allá de los contextos, situaciones aun cotidianas.

El método biográfico sirve para reconstruir prácticas de una disciplina en particular en un momento y lugar específicos. Refiriéndose al ámbito de las ciencias se dice que "biografías individuales y biografías de colectivos, elaboradas con metodologías rigurosas y fuentes creativas, construyen un espacio respetado en la historiografía de la ciencia. No se trata solo de acumular nombres de mujer sino de analizar cómo sus historias de vida configuran en cada momento histórico las prácticas científicas y con ellas la ciencia misma" (Santesmases, Cabre i Pairet y Ortiz Gómez, 2017, p. 400).

Uno de los objetivos del trabajo fue potenciar el papel de las mujeres en internet y en las redes sociales, poniendo a disposición del público material de calidad. Las plataformas de intercambio virtual que manejamos, Wordpress, Twitter y Facebook, permiten un efecto multiplicador de estos objetivos. A través de las redes se llega a un público más amplio, rompiendo las barreras de la academia.

El proyecto "Arquitectas en la web" permitió difundir el blog en nuevos contextos. Durante la vista al IAWA, Florencia Marciani fue in- 
vitada a participar del ciclo de conferencias "Fire in the Library" en la Facultad de Arquitectura de Virginia Tech. Además, en 2017 Un día| una arquitecta participó en el Simposio IAWA, con la presentación de un video que sintetizó la experiencia.

Por último, también se compilaron las 50 biografías en un documento que fue donado el IAWA para formar parte de las colecciones (Moisset, Marciani, Barraud et al., 2017). 


\section{Referencias}

Aguirre Ramírez, C. y Villa-Flores, J. (2009). Los archivos y la construcción de la verdad histórica en América Latina. Jahrbuch für Geschichte Lateinamerikas = Anuario de Historia de América Latina (JbLA), 46, 5-17.

Álvarez, E. y Gómez, C. (2017, 8 de marzo). The Invisible Women: How female architects were erased from history. [Entrada de blog] Architectural Review. Recuperado el 25 de julio de 2018, de: https:/ / www. architectural-review.com/essays/the-invisible-women-how-female-architects-were-erased-from-history/10017481.article.

Barraud, S. (2016, 27 de septiembre). Mary Colter 18691958. [Entrada de blog] Un día | Una arquitecta 2. Recuperado el 25 de julio de 2018, de: https:/ / undiaunaarquitecta2.wordpress.com/2016/09/27/ mary-colter-1869-1958/.

Bione, C. (2005). Introduzione. Parametro: Rivista Internazionale di Architettura e Urbanistica, XXXV(257), 17.

Blazquez Graf, N. (2012). Epistemología feminista: Temas centrales. En Blázquez Graf, N., Flores Palacios, F. y Ríos Everardo, M. (Eds.), Investigación Feminista: epistemología, metodología y representaciones sociales, 21-38. México: UNAM / Centro de Investigaciones Interdisciplinarias en Ciencias y Humanidades. Recuperado el 16 de julio de 2018, de: http:/ /investigacion.cephcis.unam.mx/generoyrsociales/wp-content/uploads/2016/04/Investigacion-Feminista-1.pdf.

Colectivo Un Día | Una Arquitecta (s.f.). Un Día | Una arquitecta [Blog]. Recuperado el 25 de julio de 2018, de: https://undiaunaarquitecta.wordpress. $\mathrm{com} /$.

Diez Jorge, M.E. (2014, enero-junio). Investigar sobre la arquitectura y el género: teoría y praxis de un proyecto. Arenal, 21(1), 179-190. Recuperado el 25 de julio de 2018, de: https:/ / dialnet.unirioja.es / descarga/articulo/4747168.pdf.

Gropius, W. (1956). Alcances de la arquitectura integral. Buenos Aires: La Isla.

IAWA (s.f.). Guide to the IAWA Collections. [versión electrónica]. Recuperado el 25 de julio de 2018, de: https://spec.lib.vt.edu/IAWA/guide.html

Ketelaar, E. (2007). The Panoptical Archive. En Blouin, F. X. y Rosenberg, W. G. (eds.), Archives, Documentation, and Institutions of Social Memory. Essays from the Sawyer Seminar, pp. 144-150. Ann Arbor: The University of Michigan Press.

Marciani, F. (2015, 25 de abril). Charlotte Perriand 19031999. [Entrada de blog] Un día / una arquitecta. Recuperado el 25 de julio de 2018, de: https:/ / undiaunaarquitecta.wordpress.com/2015/04/25/ charlotte-perriand-1903-1999/.

Marciani, F. (2016, 20 de noviembre). Hanna Adamc-
zewska-Wejchert 1920-1996. [Entrada de blog] Un día | una arquitecta 2. Recuperado el 25 de julio de 2018, de: https:/ / undiaunaarquitecta2.wordpress.com/2016/11/20/hanna-adamczewska-wejchert-1920-1996/.

Moisset, I. (2015, 28 de mayo). Anne Tyng 1920-2011. [Entrada de blog] Un día | una arquitecta. Recuperado el 25 de julio de 2018, de: https://undiaunaarquitecta.wordpress.com/2015/05/28/annetyng-1920-2011/.

Moisset, I. (2016, 30 de octubre). Masako Hayashi 19282001 | Hatsue Yamada 1930 | Nobuko Nakahara 19292008. [Entrada de blog] Un día | una arquitecta 2. Recuperado el 25 de julio de 2018, de: https: / / undiaunaarquitecta2.wordpress.com/2016/10/30/ masako-hayashi-1928-2001hatsue-yamada-1928nobuko-nakahara-1929-2008/.

Moisset, I. (2017, 7 de marzo). Truus Schröder 18891985. [Entrada de blog] Un día | una arquitecta 2. Recuperado el 25 de julio de 2018, de: https://undiaunaarquitecta2.wordpress.com/2017/03/07/ truus-schroder-1889-1985/.

Moisset, I., Marciani, F., Barraud, S., et al. (2017). Women architects on the web. Recuperado el 25 de julio de 2018, de: https://undiaunaarquitecta3.files. wordpress.com/2017/11/women-architects-on-theweb-iawa.pdf.

Muxí, Z. (2015, 9 de marzo). Plautilla Bricci 1616. [Entrada de blog] Un día | una arquitecta. Recuperado el 25 de julio de 2018, de: https://undiaunaarquitecta.wordpress.com/2015/03/09/plautilla-bricci-1616/.

Ojeda, G. (2015, 3 de mayo). Sadie Speight 1906-1992. [Entrada de blog] Un día | una arquitecta. Recuperado el 25 de julio de 2018, de: https:/ / undiaunaarquitecta.wordpress.com/2015/05/03/sadiespeight-1906-1992/.

Ojeda, G. (2016, 22 de octubre). Luo Xiaowei 1925. [Entrada de blog] Un día | una arquitecta 2. Recuperado el 25 de julio de 2018, de: https://undiaunaarquitecta2.wordpress.com/2016/10/22/1uoxiaowei-1925/.

Perrot, M. (2009). Mi historia de las mujeres. Buenos Aires: Fondo de Cultura Económica.

Rivera, S. (2015, 14 de junio). Milka Bliznakov 19272010. [Entrada de blog] Un día | una arquitecta. Recuperado el 25 de julio de 2018, de: https:// undiaunaarquitecta.wordpress.com/2015/06/14/milka-bliznakov-1927-2010/.

Russ, J. (1983). How to suppress womens writing. Austin: University of Texas Press.

Santesmases, M.J., Cabre i Pairet, M. y Ortiz Gomez, T. (2017). Feminismos biográficos: aportaciones 
desde la historia de la ciencia. Arenal, 24(2), 379404. Recuperado el 25 de julio de 2018, de: http:// revistaseug.ugr.es/index.php/arenal/article/ view/6303.

Zellner, P. (2016). El Archivo Internacional de Mujeres en Arquitectura celebra 30 años: una nueva vi- sión. Bitácora arquitectura, 33, pp. 16-23. Recuperado el 25 de julio de 2018, de: https:/ /biblat.unam. $\mathrm{mx} / \mathrm{ca} /$ revista/bitacora-arquitectura/2.

Zemon Davies, N. (1999). Mujeres de los márgenes: tres vidas del siglo XVII. Madrid: Cátedra.

Marciani, F. y Moisset, I. (2018). Arquitectas en la web. Hábitat y Sociedad, 11, 223-238.

<http://dx.doi.org/10.12795/HabitatySociedad.2018.i11.13>

$\bigcirc$ 